\title{
Co-pyrolysis of sewage sludge and rice straw: Thermal behavior and char characteristics evaluations
}

Qing Dong, ${ }^{*}, \dagger$ Shuping Zhang, ${ }^{*}, \AA$ Bo Wu,${ }^{\perp}$ Meng Pi, ${ }^{\ddagger}$ Y Yanquan Xiong,,${ }^{\S}$ Huiyan Zhang,,${ }^{\S}$

†School of Life Science and Food Engineering, Jiangsu Provincial Engineering Laboratory for

Biomass Conversion and Process Integration, Huaiyin Institute of Technology, Huaian,

$$
\text { 223003, Jiangsu, China }
$$

¥School of Energy and Power Engineering, Nanjing University of Science and Technology,

Nanjing 210094, Jiangsu, China

\$Key Laboratory of Energy Thermal Conversion and Control of Ministry of Education,

School of Energy and Environment, Southeast University, Nanjing 210096, Jiangsu, China

${ }^{\perp}$ Research Center of Heterogeneous Catalysis and Engineering Sciences, School of Chemical

Engineering and Energy, Zhengzhou University, Zhengzhou 450001, Henan, China

${ }^{*}$ Corresponding Author

E-mail: dongq@hyit.edu.cn (Dong Q.); zhangsp@njust.edu.cn (Zhang S.) 
Table S1 Basic fuel properties of SS and RS.

\begin{tabular}{lll}
\hline Properties & $\mathrm{SS}$ & $\mathrm{RS}$ \\
\hline Proximate analysis (dry basis, wt.\%) & & \\
Ash & 53.18 & 9.58 \\
Volatile & 44.16 & 74.14 \\
Fixed carbon & 2.66 & 16.28 \\
Elemental analysis (dry basis, wt.\%) & & \\
$\mathrm{C}$ & 18.78 & 41.25 \\
$\mathrm{H}$ & 3.71 & 5.59 \\
$\mathrm{O}$ & 21.03 & 42.3 \\
$\mathrm{~N}$ & 2.72 & 1.12 \\
$\mathrm{~S}$ & 0.58 & 0.16 \\
$\mathrm{HHV}(\mathrm{MJ} / \mathrm{kg})$ & 7.65 & 16.41 \\
$\mathrm{Ash} \mathrm{compositions} \mathrm{(wt. \% )}$ & & \\
$\mathrm{Na}_{2} \mathrm{O}$ & 4.55 & 1.61 \\
$\mathrm{~K}_{2} \mathrm{O}$ & 35.51 & 4.80 \\
$\mathrm{MgO}_{\mathrm{CaO}}$ & 2.54 & 6.51 \\
$\mathrm{MnO}_{\mathrm{Al}} \mathrm{O}_{3}$ & 0.25 & 9.67 \\
$\mathrm{Fe}_{2} \mathrm{O}_{3}$ & 0.43 & 0.05 \\
$\mathrm{SiO}_{2}$ & 2.82 & 20.91 \\
$\mathrm{P}_{2} \mathrm{O}_{5}$ & 0.25 & 11.34 \\
$\mathrm{SO}_{3}$ & 36.78 & 33.20 \\
$\mathrm{ClO}_{2}$ & 0 & 10.12 \\
$\mathrm{ZnO}_{\mathrm{PbO}}$ & 5.01 & 1.20 \\
$\mathrm{CuO}$ & 2.49 & 0.31 \\
\hline & 9.37 & 0.19 \\
& 0 & 0.05 \\
& 0 & 0.04 \\
\hline
\end{tabular}



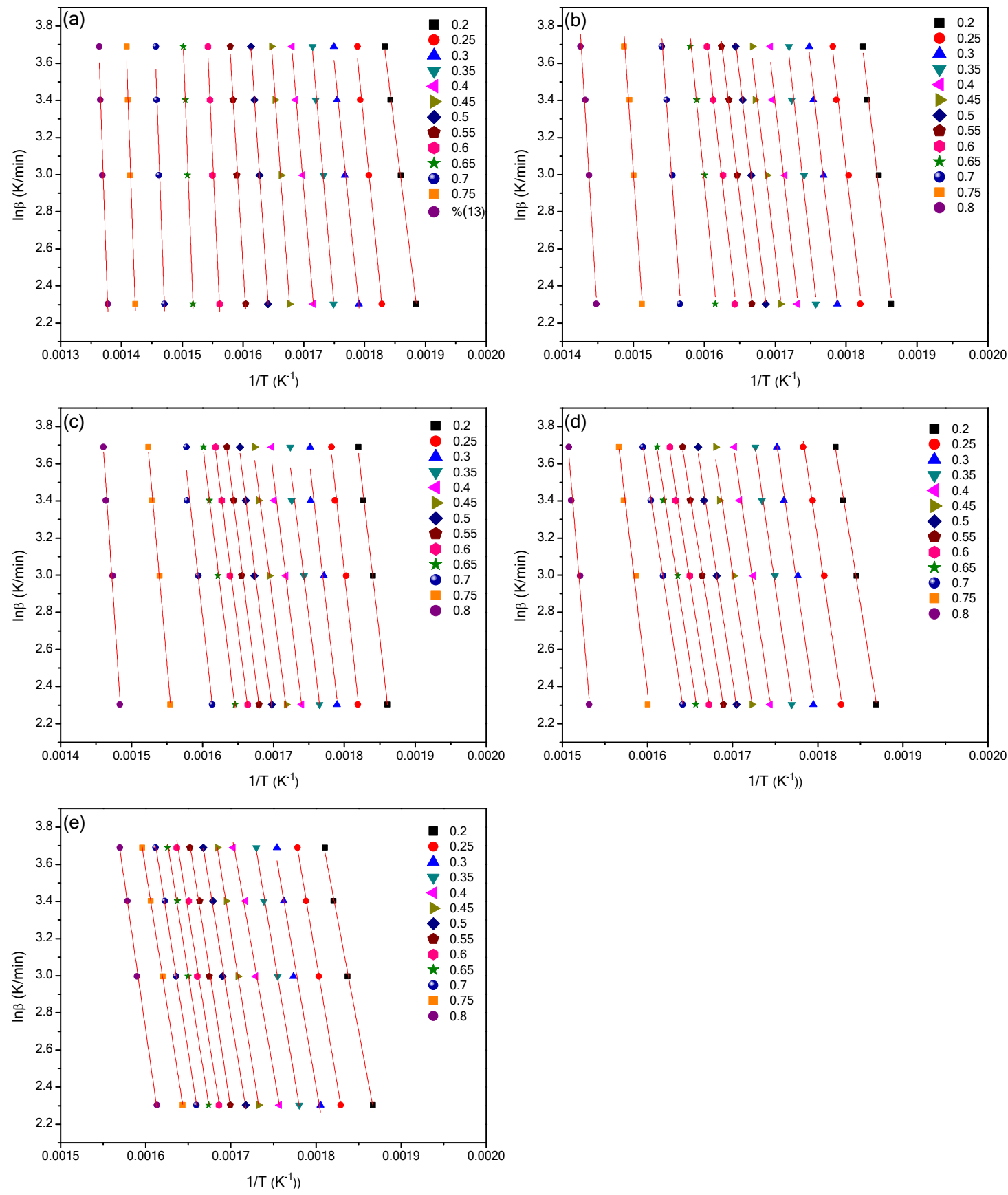

Fig. S1. Linear regression of the relationship between $\ln \beta$ and $-1 / T$ at various $\alpha$ values: (a) SS, (b) $7 \mathrm{SS} / 3 \mathrm{RS}$, (c) $5 \mathrm{SS} / 5 \mathrm{RS}$, (d) $3 \mathrm{SS} / 7 \mathrm{RS}$, (e) RS. 